\title{
Can SOCS make arthritis better?
}

\author{
Lionel B. Ivashkiv ${ }^{1,2}$ and Ioannis Tassiulas ${ }^{1}$ \\ ${ }^{1}$ Department of Medicine, Hospital for Special Surgery, New York, New York, USA \\ ${ }^{2}$ Graduate Program in Immunology, Weill Medical College of Cornell University, New York, \\ New York, USA \\ J. Clin. Invest. 111:795-797 (2003). doi:10.1172/JCI200318113.
}

Rheumatoid arthritis (RA) is a chronic inflammatory disease characterized by autoimmunity and infiltration of joint synovium by activated inflammatory cells, including neutrophils, macrophages, lymphocytes, plasma cells, and osteoclasts (reviewed in ref. 1). Morbidity in RA occurs secondary to the destruction of cartilage and bone by an inflammatory mass termed the pannus, which consists predominantly of activated macrophages and proliferating synovial fibroblasts. Synovial inflammation is regulated by cytokines (1). Pro-inflammatory cytokines implicated in RA pathogenesis include TNF- $\alpha$, IL-1, IL-6, IL-12, IL-15, IL-18, IFN- $\gamma$, and GM-CSF. A key role for TNF- $\alpha$ and IL-1 in RA pathogenesis has been confirmed by the success of TNF and IL-1 neutralization in the treatment of RA. RA synovium is also characterized by the expression of anti-inflammatory cytokines and cytokine antagonists, including TGF- $\beta$, IL-10, the IL-1 receptor antagonist, and soluble TNF receptors. Production of these factors reflects an attempt to control inflammation and achieve homeostasis. The idea that the balance between pro- and anti-inflammatory factors is important in regulating the rate of

\footnotetext{
Address correspondence to: Lionel B. Ivashkiv, Department of Medicine, Hospital for Special Surgery, 535 East 70th Street, New York, New York 10021, USA. Phone: (212) 606-1653; Fax: (212) 774-2337; E-mail: ivashkivl@hss.edu.

Conflict of interest: The authors have declared that no conflict of interest exists. Nonstandard abbreviations used: rheumatoid arthritis (RA); suppressors of cytokine signaling (SOCS); Janus kinase-signal transducers and activators of transcription (JAK-STAT); methylated BSA (mBSA); Toll-like receptor (TLR).
}

progression, and thus the eventual severity and morbidity, of RA has gained broad acceptance among RA researchers (1).

\section{Inhibition of cytokine signaling by suppressor of cytokine signaling proteins}

The current paradigm states that cytokine balance is determined by the relative levels of expression of proinflammatory and anti-inflammatory cytokines. However, cytokine action is modulated by feedback inhibition and signal transduction crosstalk among synergistic or opposing cytokines. Thus the regulation of cellular responsiveness to cytokines determines cytokine activity and the balance between opposing cytokines. Key regulators of cellular responses to cytokines are members of the suppressors of cytokine signaling (SOCS; also known as cytokine-induced $\mathrm{SH} 2$ containing protein, Jak binding protein, or STAT-induced STAT inhibitor) protein family, which plays an important role in feedback inhibition and cytokine cross-regulation $(2,3)$. SOCS were originally discovered, and are best known, as inhibitors of the Janus kinase-signal transducer and activator of the transcription (JAKSTAT) signaling pathway. SOCS inhibit JAK-STAT signaling by: (i) inhibiting JAK catalytic activity; (ii) competing with STATs for receptor docking sites, and (iii) targeting cytokine receptors for degradation by proteosomes. SOCS-1, SOCS-2, SOCS-3, and cytokine-induced $\mathrm{SH} 2$-containing protein expression is rapidly induced by many cytokines, including cytokines that activate the JAK-STAT pathway. Individual SOCS are capable of inhibiting multiple cytokines, and it is not clear how the specificity of inhibition by SOCS is regulated.

\section{JAK-STAT signaling and inflammatory arthritis}

STATs are latent cytoplasmic proteins that are activated by tyrosine phosphorylation, which leads to dimerization, acquisition of DNA binding activity, and translocation to the nucleus and transcriptional activation of genes (4). Several cytokines implicated in RA pathogenesis activate STATs, namely, IL- 6 family cytokines (activate STAT3), IL-12 (STAT4), IL-15 (STAT3 and STAT5), GM-CSF (STAT5), and IFN- $\gamma$ (STAT1). STAT1 and STAT4 expression is elevated, and STAT 1 and STAT3 are activated, in the synovium in RA and experimental arthritis (refs. 5-8, L. Ivashkiv and A. Koch, unpublished observations). Ablation of STAT3 activity using a dominant negative STAT3 mutant results in apoptosis of synovial fibroblasts (9), and gene transfer of a dominant negative STAT3 mutant to synovial macrophages and fibroblasts attenuates collagen-induced arthritis (6), demonstrating a role for STAT3 in the pathogenesis of inflammatory arthritis. Overexpression of SOCS-3 in joint macrophages and fibroblasts has been demonstrated to effectively block STAT3 activation during experimental arthritis and to attenuate disease severity (6). Thus, SOCS-3 overexpression is effective in attenuating arthritis, but the role of endogenous SOCS expression and the mechanisms by which SOCS-3 overexpression suppresses arthritis have not been elucidated. The paper by Egan and colleagues in this issue of the JCI (10) investigates the role of endogenous SOCS- 1 in modulating acute arthritis induced by the injection of methylated BSA and IL-1 (mBSA/IL-1 arthritis).

\section{SOCS-1 and arthritis}

mBSA/IL-1 arthritis is an acute arthritis that peaks seven days after induction and resolves by day 21 . Similarities to RA include infiltration of joints by myeloid cells, pannus formation, and a combination of inflammation and tissue destruction. mBSA/IL-1 arthritis is independent of $B$ cells or $\mathrm{CD}^{+} \mathrm{T}$ cells, but is dependent upon 


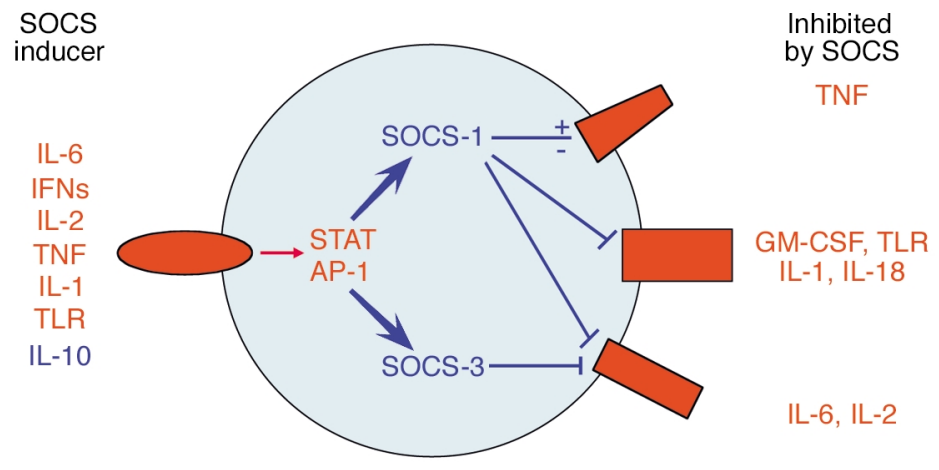

Figure 1

Modulation of cytokine signaling by SOCS proteins during inflammatory arthritis. Multiple cytokines can induce SOCS expression, which subsequently inhibits signaling by many of the same cytokines. Feedback inhibition and cross-regulation of signaling contribute to suppression of cytokine activity and attenuation of inflammatory arthritis. SOCS expression is induced as part of a feedback inhibition loop by cytokines that are pro-inflammatory (shown in red) or by anti-inflammatory cytokines such as IL-10 (shown in blue). AP-1, activator protein-1.

$\mathrm{CD}^{+} \mathrm{T}$ cells, and is modulated by TNF- $\alpha$, GM-CSF, and IL- 6 . The authors found that mBSA/IL-1 arthritis was independent of IFN- $\gamma$. Mechanisms by which $\mathrm{CD}^{+} \mathrm{T}$ cells drive inflammatory arthritis and synovial infiltration and activation of macrophages in the absence of IFN- $\gamma$ may be important in RA pathogenesis (11) and can be delineated using this model. The independence of mBSA/ IL- 1 arthritis from IFN- $\gamma$ allowed the authors to address the role of SOCS-1 using SOCS-1-/- IFN- $\gamma^{-/-}$doubleknockout mice, which do not develop the severe spontaneous inflammation and perinatal lethality manifested by SOCS-1 $1^{-/-}$mice (12-14). Deficiency of SOCS-1 resulted in increased severity but no change in the time course of arthritis. SOCS-1/- IFN- $\gamma^{-/-}$arthritic mice exhibited increased myeloid cell infiltration of synovium and adenopathy of draining lymph nodes, with hyperproliferation of T cells. Immunohistochemistry, using a SOCS-1 antibody and measuring the activity of a lac $Z$ reporter gene that was integrated into the SOCS-1 locus, indicated that the SOCS-1 gene was expressed in synovial macrophages, lymphocytes, and fibroblasts, and in activated lymph node $T$ cells, during the course of mBSA/IL-1 arthritis.

These results clearly identify SOCS1 as an important negative regulator of acute arthritis in the $\mathrm{mBSA} / \mathrm{IL}-1$ arthritis model. Increasing SOCS-1 expression in the appropriate cells at the appropriate time may represent a promising new approach to the treatment of inflammatory arthritis. This study also raises a number of interesting questions, several of which can be readily addressed in the mBSA/IL-1 arthritis model. One important question is in which cells and tissues SOCS-1 exerts its antiarthritic activity. Previous work showed that SOCS-1/- lymphocytes are both necessary and sufficient for the spontaneous lethal inflammatory syndrome manifested by SOCS $-1^{-/-}$ mice (14). Thus it is likely that suppression of $T$ cell expansion and activation in draining lymph nodes is an important mechanism of SOCS-1 suppression of mBSA/IL-1 arthritis. This represents an interesting contrast to SOCS-3, which suppresses arthritis when overexpressed in synovial macrophages and fibroblasts (6). Thus, different SOCS proteins may suppress different components of autoimmunity and inflammation that are important in arthritis.

Another important question is which cytokine(s) need to be inhibited by SOCS-1 in order to attenuate arthritis? SOCS proteins are best known as inhibitors of JAK-STAT signaling, but they also interact with the adaptor protein Grb2, the phosphoprotein Vav, calcineurin, and IL-1 receptor-associated kinase, and thereby suppress signaling by Kit, the $\mathrm{T}$ cell antigen receptor, and Toll-like receptor 4 (TLR4), which shares a major signaling pathway with IL-1 (15-18). In addition, SOCS can potentiate Ras-MAPK pathways and suppress TNF-induced apoptosis $(19,20)$. Thus, SOCS-1 may suppress signaling by many different cytokines in mBSA/IL-1 arthritis (see Figure 1), and the cytokines that are suppressed will vary according to cell type and inflammatory microenvironment. In the draining lymph nodes, SOCS-1 most likely functions by suppressing signaling by cytokines important for $\mathrm{T}$ cell survival and proliferation, such as IL-6 and IL-2. In the synovium, SOCS- 1 could potentially suppress signaling by multiple pro-inflammatory cytokines, including endogenous IL-1, IL-18, IL-6, GM-CSF, and endogenous TLR ligands, and could alter TNF signaling. In addition, because SOCS-1 binds to IL-1 receptor-associated kinase and suppresses TLR pathways that are also used by IL-1 $(17,18)$, SOCS-1 can inhibit the action of exogenous IL-1 during induction of mBSA/IL-1 arthritis. In summary, SOCS-1 may suppress inflammatory arthritis by inhibiting signaling by multiple cytokines.

SOCS-1 deficiency results not only in enhanced cellular responses to cytokines, but also in increased cytokine production in vivo, including elevated basal serum levels of IFN- $\gamma$ and TNF- $\alpha(14,19)$. Although Egan et al. (10) did not detect any changes in IL-1, IL-6, or IL-18 mRNA levels in synovium in SOCS-1-/- IFN- $\boldsymbol{\gamma}^{-/-}$arthritic mice relative to control arthritic mice, it will be important to carefully examine the effects of SOCS-1 deficiency on cytokine production in both synovium and draining lymph nodes. It is also interesting to speculate about which cytokines induce SOCS-1 expression during arthritis (Figure 1). Possibilities that come to mind include IL- 6 and IL-10 in T cells in lymph nodes $(21,22)$, and IL-6, IL-10, TNF, IL-1, and endogenous TLR ligands in synovium. Of note, most of these are pro-inflammatory cytokines that induce SOCS expression as part of feedback inhibition, except for IL-10 that is antiinflammatory. The roles of various cytokines in the induction of SOCS expression can be addressed using appropriate knockout mice.

In summary, this interesting paper (10) identifies SOCS-1 as an important suppressor of acute inflammatory arthritis and suggests novel approaches to arthritis therapy based on increasing SOCS-1 expression. The important role of SOCS- 1 in modulating arthritis severity emphasizes the 
importance of feedback inhibition and signal transduction cross-regulation in synovial inflammation. Further study of mechanisms by which SOCS proteins regulate inflammatory arthritis are likely to yield important insights into RA pathogenesis and cytokine regulatory networks in complex inflammatory diseases.

1. Feldmann, M., Brennan, F.M., and Maini, R.N. 1996. Role of cytokines in rheumatoid arthritis. Annu. Rev. Immunol. 14:397-440.

2. Yasukawa, H., Sasaki, A., and Yoshimura, A. 2000. Negative regulation of cytokine signaling pathways. Annu. Rev. Immunol. 18:143-164.

3. Nicola, N.A., et al. 1999. Negative regulation of cytokine signaling by the SOCS proteins. Cold Spring Harb. Symp. Quant. Biol. 64:397-404.

4. Schindler, C., and Darnell, J.E., Jr. 1995. Transcriptional responses to polypeptide ligands: the JAK-STAT pathway. Annu. Rev. Biochem. 64:621-651

5. Wang, F., Sengupta, T.K., Zhong, Z., and Ivashkiv, L.B. 1995. Regulation of the balance of cytokine production and the signal transducer and activator of transcription (STAT) transcription factor activity by cytokines and inflammatory synovial fluids. J. Exp. Med. 182:1825-1831.
6. Shouda, T., et al. 2001. Induction of the cytokine signal regulator SOCS3/CIS3 as a therapeutic strategy for treating inflammatory arthritis. J. Clin. Invest. 108:1781-1788. doi:10.1172/JCI200113568.

7. Frucht, D.M., et al. 2000. Stat4 is expressed in activated peripheral blood monocytes, dendritic cells, and macrophages at sites of Th1-mediated inflammation. J. Immunol. 164:4659-4664.

8. Hu, X., et al. 2002. Sensitization of IFN-gamma Jak-STAT signaling during macrophage activation. Nat. Immunol. 3:859-866.

9. Krause, A., Scaletta, N., Ji, J.D., and Ivashkiv, L.B. 2002. Rheumatoid arthritis synoviocyte survival is dependent on Stat3. J. Immunol. 169:6610-6616

10. Egan, P.J., Lawlor, K.E., Alexander, W.S., and Wicks, I.P. 2003. Suppressor of cytokine signaling-1 regulates acute inflammatory arthritis and $\mathrm{T}$ cell activation. J. Clin. Invest. 111:915-924. doi:10.1172/JCI200316156.

11. Firestein, G.S., and Zvaifler, N.J. 2002. How important are $T$ cells in chronic rheumatoid synovitis? II. T cell-independent mechanisms from beginning to end. Arthritis Rheum. 46:298-308.

12. Naka, T., et al. 1998. Accelerated apoptosis of lymphocytes by augmented induction of Bax in SSI-1 (STAT-induced STAT inhibitor-1) deficient mice. Proc. Natl. Acad. Sci. U. S. A. 95:15577-15582.

13. Alexander, W.S., et al. 1999. SOCS1 is a critical inhibitor of interferon gamma signaling and prevents the potentially fatal neonatal actions of this cytokine. Cell. 98:597-608.
14. Marine, J.C., et al. 1999. SOCS1 deficiency causes a lymphocyte-dependent perinatal lethality. Cell. 98:609-616.

15. De Sepulveda, P., et al. 1999. Socs 1 binds to mul tiple signalling proteins and suppresses steel factor-dependent proliferation. EMBOJ. 18:904-915.

16. Banerjee, A., Banks, A.S., Nawijn, M.C., Chen, X.P., and Rothman, P.B. 2002. Cutting edge: Suppressor of cytokine signaling 3 inhibits activation of NFATp. J. Immunol. 168:4277-4281.

17. Kinjyo, I., et al. 2002. SOCS1/JAB is a negative regulator of LPS-induced macrophage activation. Immunity. 17:583-591.

18. Nakagawa, R., et al. 2002. SOCS-1 participates in negative regulation of LPS responses. Immunity. 17:677-687.

19. Morita, Y., et al. 2000. Signals transducers and activators of transcription (STAT)-induced STAT inhibitor-1 (SSI-1)/suppressor of cytokine signaling-1 (SOCS-1) suppresses tumor necrosis factor alpha-induced cell death in fibroblasts. Proc Natl. Acad. Sci. U. S. A. 97:5405-5410.

20. Cacalano, N.A., Sanden, D., and Johnston, J.A. 2001. Tyrosine-phosphorylated SOCS-3 inhibits STAT activation but binds to p120 RasGAP and activates Ras. Nat. Cell. Biol. 3:460-465.

21. Diehl, S., et al. 2000. Inhibition of Th1 differentiation by IL- 6 is mediated by SOCS1. Immunity. 13:805-815

22. Ding, Y., et al. 2003. Suppressor of cytokine signaling 1 inhibits IL-10-mediated immune responses. J. Immunol. 170:1383-1391.

\title{
Autonomic "myasthenia": the case for an autoimmune pathogenesis
}

\author{
Daniel B. Drachman
}

Department of Neurology and Neuroscience, Johns Hopkins School of Medicine, Baltimore, Maryland, USA

J. Clin. Invest. 111:797-799 (2003). doi:10.1172/JCI200318180.

Of all neurological diseases, the one that virtually all third year medical students get right on final exams is myasthenia gravis (MG). MG is an autoimmune disorder that causes weakness and fatigue of skeletal muscles due to an antibody-mediated

\footnotetext{
Address correspondence to: Daniel B. Drachman, Department of Neurology and Neuroscience, Johns Hopkins School of Medicine, 600 North Wolfe Street, Baltimore, Maryland 21287-7519, USA.

Phone: (410) 955-5406; Fax: (410) 955-1961;

E-mail: dandrac@aol.com.

Conflict of interest: The author has declared that no conflict of interest exists.

Nonstandard abbreviations used: myasthenia gravis (MG); autoimmune autonomic neuropathy (AAN); musclespecific kinase (MuSK)
}

attack directed against AChRs at neuromuscular junctions (1). It is easy to learn and remember the pathogenesis, immunology, and treatment of MG because the pieces of the puzzle fit together nearly perfectly. In this issue of the JCI, Lennon et al. now provide intriguing evidence of another putative autoimmune disease of AChRs - autoimmune autonomic neuropathy (AAN) (2). They have shown that immunization of rabbits with a fragment of ganglionic AChR induces a condition that mimics the human disease. AAN is very rare but can be life threatening. It is manifested by autonomic disturbances affecting the sympathetic and parasympathetic nervous systems: abnormal gastrointestinal motility with stalled traffic in the intestines, impaired contraction and striking dilatation of the bladder, and disturbances of blood pressure, sweating, salivation, pupillary reactions, and sexual function (3). Based on these experiments and other evidence (4), Lennon et al. suggest that: (a) an antibody-mediated attack directed against neuronal AChRs of autonomic ganglia may be implicated in at least some cases of AAN; and (b) the autoimmune response in some of these patients may be triggered by a remote neoplasm that incidentally expresses the autoantigen. These new findings provide interesting insights into the concepts of autoimmune and paraneoplastic diseases of the nervous system, and information that may be useful for practical treatment of AAN.

\section{Five criteria for recognizing antibody-mediated autoimmune disease}

The list of candidate autoimmune diseases affecting every level of the nervous system is long and rapidly increasing. However, it is not simple to prove that a given disease is autoimmune in nature, and even more difficult to identify the autoimmune mechanisms and molecular 